\title{
Growth and characterization of a new NLO material: 4-methoxy benzoin
}

\author{
B. Thanuja ${ }^{1}$, G. Nithya ${ }^{1}$, C. Kanagam ${ }^{2}$ \\ ${ }^{I}$ Department of Chemistry, Vels University, School of Engineering, Chennai-600 117, India; \\ ${ }^{2}$ Department of chemistry, SRM Vallimmai Engineering college, Kattangalathur, India.
}

Received January 29, 2012; accepted March 30, 2012; published March 31, 2012

\begin{abstract}
Single crystals of 4-methoxy benzoin (4MB), an organic nonlinear optical material have been synthesized by a slow evaporation technique at room temperature. The crystalline nature was confirmed by single crystal X-ray diffraction analysis. The chemical composition of grown crystals was determined by FTIR spectra. Optical absorption studies have shown that UV cut off wavelength of the grown crystal is at $400 \mathrm{~nm}$ and it has a wide transparency window. The second harmonic generation (SHG) efficiency of the grown crystal was confirmed by the Kurtz and Perry powder technique.
\end{abstract}

Highly nonlinear materials are capable of fast and efficient processing of optical signals in the fast growing development of optical fiber communication systems. The nonlinear crystals have proved to be interesting candidates for a number of applications, such as second harmonic generation, frequency mixing, electro-optic modulation, optical parametric oscillation etc. [1]. Due to the technological importance of these nonlinear crystals the needs for high quality organic crystals have grown dramatically in the last decade. With rapid progress in the crystal growth technology, crystals having attractive nonlinear properties are being discovered. This has enabled the commercial development of single crystals with promising nonlinear optical properties. An organic molecule with significant nonlinear optical activity generally consists of a $\pi$-electron conjugated moiety substituted by an electron donor group at one end of the conjugated structure and an electron acceptor at the other end, forming a "push-pull" conjugated structure. The conjugated $\pi$-electron moiety provides a pathway for the entire length of conjugation under the perturbation of an external electric field. Functionalizing both ends of the conjugated $\pi$ bond system with an appropriate electron donor and acceptor group can increase the asymmetric electronic distribution in either or both the ground and excited state, thus leading to increased optical nonlinearity [2]. Organic molecules should have high second order hyperpolarizability $(\beta)$ to exhibit effective NLO properties in many cases, an order of magnitude smaller than widely known inorganic materials [3].

4-methoxy benzoin is a new potential candidate for second order NLO applications. The molecular design of 4-methoxy benzoin, containing one electron donor (methoxy) and one electron acceptor (carbonyl) moiety, provides it with a push-pull configuration, which is a well-known way of enhancing optical nonlinearities. In this paper we report the growth, structural, vibrational, optical and second harmonic generation $(\mathrm{SHG})$ properties of single crystals of 4-methoxy benzoin(4MB).

The title compound was synthesized by benzoin condensation using $4 \mathrm{~g}$ of $\mathrm{KCN}$ dissolved in $75 \mathrm{cc}$ of water in a one litre flask. To this was added $6.8 \mathrm{~g}(0.05 \mathrm{~mole})$ of $\mathrm{p}$-anisaldehyde, $7 \mathrm{~g}(0.05 \mathrm{~mole})$ of benzaldeyde and $75 \mathrm{cc}$ of $95 \%$ ethanol. The mixture formed a solution at the boiling temperature and was refluxed for one and half hours. Steam was then passed through the solution until all the alcohol and nearly all the unchanged aldehyde was removed. The condensed water was decanted from the product and later set aside for crystallization. The product was then pressed as free as possible from oily material on a suction funnel and washed with cold alcohol. In this way about $8.1 \mathrm{~g}$ (yield was $65 \%$ ) of crude product was obtained. The crude mixture was dissolved in alcohol and allowed to crystallize slowly. The 4-methoxy benzoin crystallized out as lumps of long needles. The melting point of the compound was found to be $110^{\circ} \mathrm{C}$.

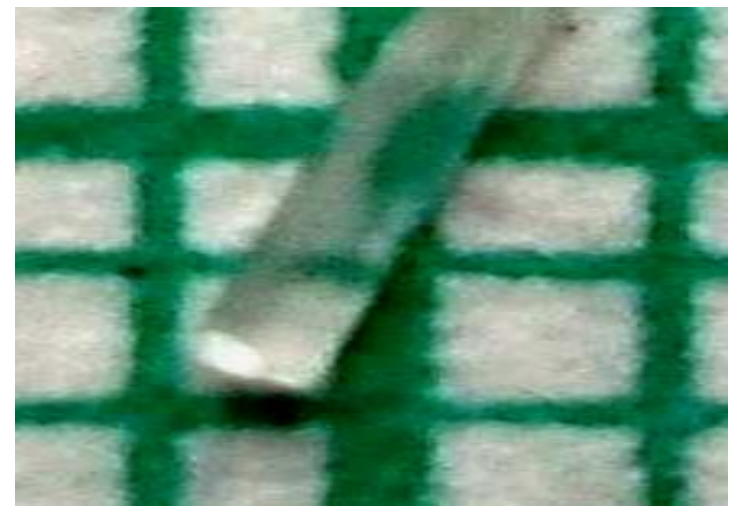

Fig.1.Single crystal X-ray diffraction.

The synthesized compound was purified by a successive recrystallization process. The melting point of the compound was found to be $110 \pm 1{ }^{\circ} \mathrm{C}$. In order to improve the purity of the synthesized compound, the basic material was purified thoroughly. The purification 
of $4 \mathrm{MB}$ was done by repeated crystallization and the purity of the material was monitored by measuring its melting point each time. After 4 weeks, single crystals with dimensions $10 \mathrm{~mm} \times 3 \mathrm{~mm} \times 2 \mathrm{~mm}$ were harvested from the mother solution by a solvent evaporation method with a growth rate of $0.4 \mathrm{~mm} /$ day. Then the purity of the crystal was improved by the successive recrystallization process. The single crystal of $4 \mathrm{MB}$ is shown in Fig. 1.

Single crystal X-ray diffraction data were recorded using a BRUKER AXS KAPPA APEX (II) CCD X-ray diffractometer with $\operatorname{MoK} \alpha$ radiation $(\lambda=0.71073 \AA)$ to identify the structure of the grown crystal. The structure was solved by a direct method and refined by the full matrix least square technique using the SHELXL program. The calculated lattice parameters are presented in Table 1. It is observed from the XRD analysis that the $4 \mathrm{MB}$ crystal has as orthorhombic structure with the space group pca $2_{1}$.

Table 1. Single crystal XRD data of 4MB crystal

\begin{tabular}{|l|l|}
\hline Unit cell dimensions & $\begin{array}{l}\mathrm{a}=14.4691(10) \mathrm{A} \\
\mathrm{b}=14.0978(10) \mathrm{A} \\
\mathrm{c}=5.8468(4) \mathrm{A}\end{array}$ \\
& $\alpha=\beta=\gamma=90^{0}$. \\
& $1192.64(14) \AA^{3}$ \\
Volume & $4,1.349 \mathrm{mg} / \mathrm{m}^{3}$ \\
Z, Calculated density & Orthorhombic, Pca21 \\
Crystal system, space & Full-matrix least-squares on \\
group Refinement method & $\mathrm{F}^{\wedge} 2$ \\
\hline
\end{tabular}

The FTIR spectrum of the title compound shows the presence of a secondary alcoholic group with broad band around $3500 \mathrm{~cm}^{-1}$ attributed to the $\mathrm{O}-\mathrm{H}$ stretching modes. The bands around $2970 \mathrm{~cm}^{-1}$ in FTIR are assigned to the aromatic $\mathrm{C}-\mathrm{H}$ stretching modes. The aliphatic $-\mathrm{CH}$ stretching vibration is assigned to the band at $2681 \mathrm{~cm}^{-1}$ and $2613 \mathrm{~cm}^{-1}$ in FTIR with weak intensity. The symmetric stretching mode of the carbonyl group appears at $1710 \mathrm{~cm}^{-1}$ with very strong intensity in the FTIR spectrum. The aromatic $\mathrm{C}=\mathrm{C}$ symmetric stretching vibrations appear at $1621 \mathrm{~cm}^{-1}$ as a very strong intensity in FTIR. The in-plane deformation of $\mathrm{C}-\mathrm{OH}$ appears around $1300-1297 \mathrm{~cm}^{-1}$ as a medium band in the FTIR. The $\mathrm{OH}$ out of plane deformation is observed as strong bands around $1000 \mathrm{~cm}^{-1}$ in FTIR. The band at $1082 \mathrm{~cm}^{-1}$ and $1078 \mathrm{~cm}^{-1}$ in FTIR is assigned to the benzene ring deformation. The band at $702 \mathrm{~cm}^{-1}$ in FTIR established the presence of a disubstituted benzene ring. The resulting spectrum is shown in Fig.2. The presence of various absorption in the FTIR of the grown crystal is in very good agreement with those of parent benzoin [4].

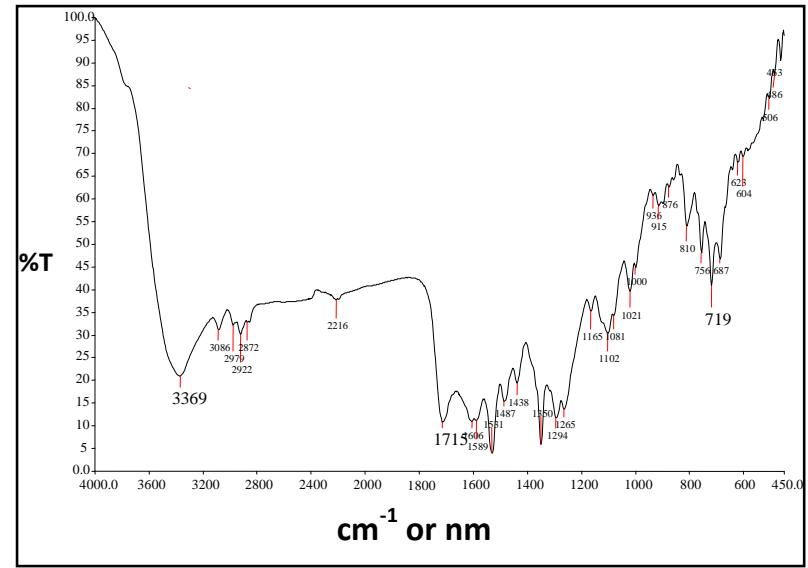

Fig. 2. FTIR spectrum of grown crystal.

The optical absorption spectrum of a good quality grown crystal was recorded in the wavelength range $200-$ 1000nm using a Cary 5E UV-vis-NIR spectrometer. The obtained absorption spectrum is shown in Fig. 3, where the lower cut off region is obtained at $240 \mathrm{~nm}$. The UV spectra show the presence of a wide transparency window lying between $400 \mathrm{~nm}$ and $1000 \mathrm{~nm}$ with $\lambda_{\max }=400 \mathrm{~nm}$. Hence, from the analysis of the absorption spectrum, it is evident that the grown crystal is transparent in the entire visible region without any absorption peak within the range, which is the key requirement for any nonlinear optical crystal.

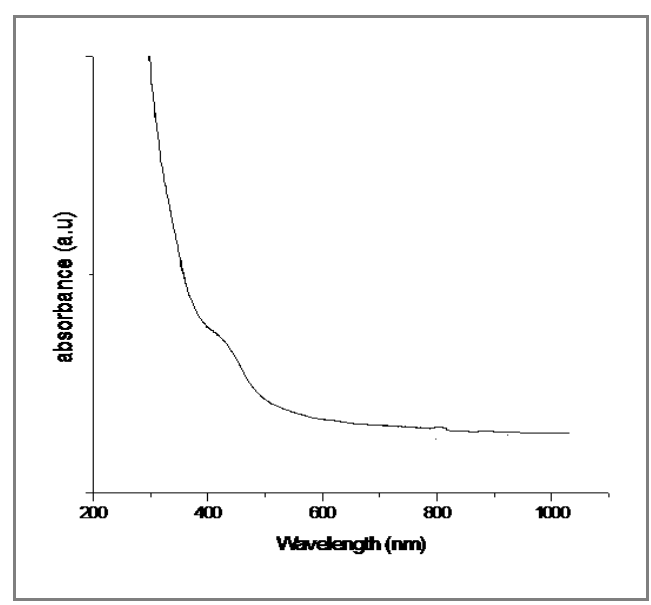

Fig. 3. Absorption spectrum of $4 \mathrm{MB}$ crystal.

In order to confirm the NLO behaviour in the title compound, powdered samples were subjected to the Kurtz and Perry powder technique [5]. A Q-switched Nd:YAG laser beam of wavelength $1064 \mathrm{~nm}$ and $10 \mathrm{~ns}$ pulse width with an input rate of $10 \mathrm{~Hz}$ was used to test the NLO property of the sample. A grown crystal SHG signal of $100 \mathrm{mV}$ was obtained while the KDP gave an SHG signal of $25.6 \mathrm{mV}$ (4 times that of KDP) for an input 
beam energy of $6.1 \mathrm{~mJ} / \mathrm{Pulse}$. The second harmonic signal generated in the crystalline sample was confirmed from the emission of green radiation from the crystal. Hence, it can be used for applications in photonic and optoelectronic devices.

In conclusion, bulk size transparent single crystals of 4MB were successfully synthesized by a slow solvent evaporation method at ambient environment. The unit cell parameters were found by single crystal and powder XRD analysis. The presence of various functional groups of the grown crystal was confirmed by FTIR. The UV-vis-NIR absorption spectra showed that $4 \mathrm{MB}$ crystal was transparent in the entire visible region with lower cut-off at $240 \mathrm{~nm}$, making it a potential candidate for NLO applications. The relative SHG conversion efficiency of the grown crystals is about 4 times higher than that of KDP sample, which indicates the suitability of $4 \mathrm{MB}$ crystals for photonic and NLO applications.
The authors are sincerely thankful to the SHG measurement facility extended by Prof. P.K. Das (Indian Institute of Science, Bangalore). The authors also acknowledge Sophisticated Analytical Instrument Facility (SAIF), Indian Institute of Technology, Chennai; Dr. M. Anbucheziyan (Dept. of Physics, SRM Valliammai Engineering College) for useful discussion. The authors acknowledge Vels University for providing the lab facilities and for giving the opportunity to do the research work. CCDC 816871 contains the supplementary crystallographic data for this paper.

\section{References}

[1] D.S. Chemla, J.Zyss, Nonlinear optical properties of organic molecules and crystals (Academic Press 1987).

[2] H. Ringertz, Acta Crystallogr. B 27, 285 (1971).

[3] T. Pal, T. Karand, B. Gabriel, L. Rigi, Cryst. Growth Des. 4, 43 (2004).

[4] S. Kashino and M. Haisa, Acta Cryst. C 41, 1066 (1985).

[5] S.K. Kurtz, T.T. Perry, J. Appl. Phys. 39, 3798 (1968). 